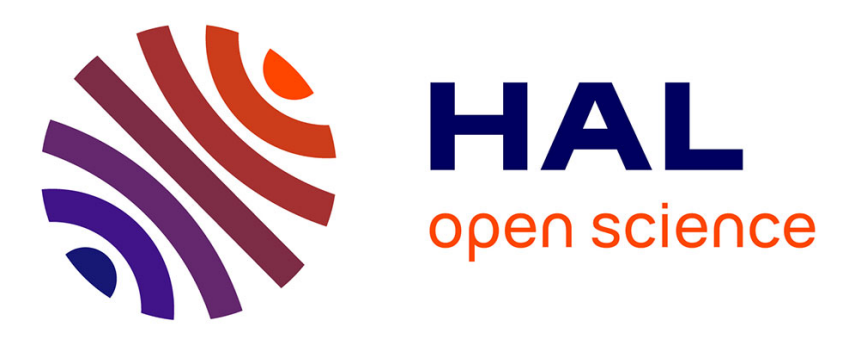

\title{
Modélisation physique du couplage des structures vibrantes dans les instruments de musique à structure complexe
}

Claude Cadoz, Olivier Grosjean

\section{- To cite this version:}

Claude Cadoz, Olivier Grosjean. Modélisation physique du couplage des structures vibrantes dans les instruments de musique à structure complexe. Journal de Physique IV Proceedings, 1994, 04 (C5), pp.C5:601-604. 10.1051/jp4:19945128 . jpa-00252804

\section{HAL Id: jpa-00252804 https://hal.science/jpa-00252804}

Submitted on 1 Jan 1994

HAL is a multi-disciplinary open access archive for the deposit and dissemination of scientific research documents, whether they are published or not. The documents may come from teaching and research institutions in France or abroad, or from public or private research centers.
L'archive ouverte pluridisciplinaire $\mathbf{H A L}$, est destinée au dépôt et à la diffusion de documents scientifiques de niveau recherche, publiés ou non, émanant des établissements d'enseignement et de recherche français ou étrangers, des laboratoires publics ou privés. 


\title{
Modélisation physique du couplage des structures vibrantes dans les instruments de musique à structure complexe
}

\author{
C. CADOZ et O. GROSJEAN
}

ACROE, 46 av. Félix Viallet, 38000 Grenoble, France

\begin{abstract}
In an instrument such as the piano, the coupling phenomena between the vibrating structures (the strings) play an important part. Firstly when there are two or three strings for each note, the coupling between these strings produces an "aftersound" phenomenon. Secondly, the vibration of the bridge causes the vibration of the other free strings. In this work, the coupling phenomena are physically modelled and simulated. The design tool is the CORDIS-ANIMA system developped by the ACROE laboratory. CORDIS-ANIMA is a digital instrument simulator based on the principle of concentrated masses (a discrete network of masses springs and dampers). At first was elaborated a model of two coupled strings as close as possible to the coupled strings in the piano. Afterwards this model was generalized to $\mathrm{N}$ strings. This new model produced aftersound as for the previous model ; but in addition it also accounted for the vibration transmission to harmonic strings. Finally, other structures than strings were also modelled on this coupling structure.
\end{abstract}

\section{INTRODUCTION}

Dans le domaine de la synthèse sonore, les deux procédés les plus courants sont les synthèses analogique et numérique. Pour ces deux méthodes, on produit (ou on reproduit) une onde sonore à partir d'oscillateurs analogiques ou numériques. La synthèse par modélisation physique propose une autre approche : il s'agit cette fois de synthétiser non plus l'onde sonore mais sa cause, c'est à dire l'instrument de musique, en tant qu'objet physique. L'intérêt d'une telle démarche est de créer des sons correspondants à une réalité physique. Le laboratoire de l'ACROE développe depuis 1979 un système de simulation : le système CORDIS, fondé sur la décomposition de tout objet physique en composants mécaniques élémentaires, la simulation de ces composants et la reconstitution des objets complexes. Dans les instruments de musique faisant intervenir plusieurs structures vibrantes, il se passe de nombreux phénomènes de couplage qui modifient sensiblement la couleur du son et sa dynamique. A l'heure actuelle, les pianos numériques se contentent de reproduire le son échantillonné de telle ou telle note, indépendamment des notes voisines. Une oreille exercée dira que le plus souvent que ces instruments numériques manquent "d'unité". Nous expliquerons sommairement dans la première partie de cet article les principes de base de CORDIS-ANIMA. Nous étudierons ensuite à travers l'exemple du piano, quels sont les phénomènes de couplage qui interviennent au niveau des structures vibrantes. Dans la troisième partie, nous expliquerons comment nous avons modélisé les mécanismes de couplage, et nous verrons quelles sont les performances du modèle.

\section{LE SYSTEME CORDIS-ANIMA.}

C'est un système de modélisation et de simulation numérique d'objets physiques audibles, visibles et manipulables [1], [2]. Les objets CORDIS qui servent à modéliser les objets physiques et qui préfigurent les algorithmes de simulation sont dotés de points de connexions de deux types, les points $L$ et les points $M$ par lesquels ils échangent des données de types forces et positions. Un point $\mathrm{L}$ produit une force et utilise une position, un point $M$ produit une position et consomme une force. La construction d'un objet CORDIS-ANIMA consiste à connecter les point $\mathrm{L}$ et $\mathrm{M}$ d'objets plus élémentaires. Tout point $\mathrm{L}$ doit être connecté à un point $\mathrm{M}$ et un seul, il y a alors sommation des forces à l'entrée des point $\mathrm{M}$ et diffusion des positions (figure 1). 


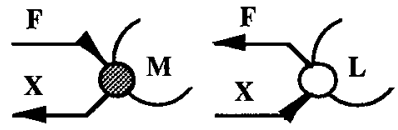

(a)

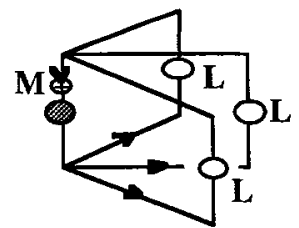

(b)

Figure 1. Représentation des points $\mathrm{M}$ et $\mathrm{L}$ (a) et règle de connexion (b).

Afin de permettre la construction d'une grande variété d'objets, CORDIS-ANIMA propose une base de modules élémentaires constituée d'un atome à deux points $\mathrm{L}$, appelé "élément de liaison", et d'un atome à un point M, appelé "point matériel" (figure 2). L'élément de liaison, produit deux forces à destination des deux points matériels auxquels il est connecté. Ces deux forces sont coaxiales, égales en intensité et de sens opposé, afin de respecter le principe d'action réaction.

L'usage typique de l'élément "point matériel" est la représentation de l'inertie localisée alors que "l'élément de liaison" représente l'interaction entre masses ponctuelles. Les trois éléments fondamentaux du système sont alors la masse, la liaison élastique, la liaison de frottement visqueux.

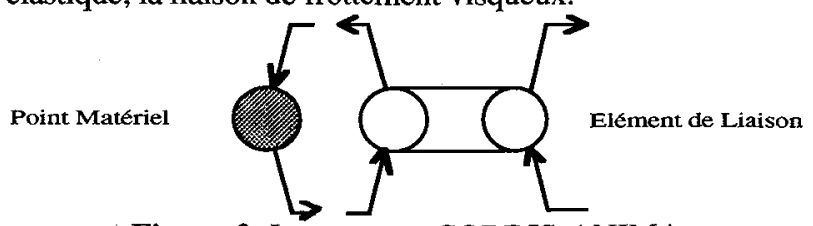

Figure 2. Les atomes CORDIS-ANIMA.

Pour obtenir un son d'un objet CORDIS, il suffit d'exciter celui-ci en déplaçant une ou plusieurs masses de leur position d'équilibre, puis de recueillir l'amplitude du déplacement d'une ou plusieurs masses de l'objet. Le signal numérique obtenu est converti en signal analogique pour être envoyé sur un sysème de haut-parleurs.

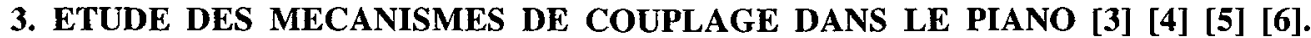

Le chevalet, dont le rôle est de transmettre les vibrations des cordes à la table d'harmonie, cède aussi de l'énergie vibratoire à toutes les cordes du piano. Plus précisément, on peut distinguer deux sortes de couplage par le biais du chevalet : d'une part une action sur les cordes d'un même doublet ou triplet (produisant la même note, mais légèrement désaccordées), d'autre part une action "lointaine" sur les cordes non percutées (vibrations par "sympathie").

\subsection{Couplage de deux cordes.}

Le son de piano évolue suivant deux régimes bien distincts. Dans un premier temps à niveau élevé, la décroissance exponentielle est très rapide. Puis apparait un changement de pente (en échelle logarithmique), indiquant un nouveau régime d'amortissement, toujours exponentiel, mais nettement moins fort. Ces deux régimes sont qualifiés d'immédiat, et de rémanent. Le phénomène est une conséquence des défauts de surface des marteaux qui produisent l'excitation faible de modes peu couplés à la table et donc peu amortis. Il s'agit des modes transversaux (déplacement orthogonal à celui du marteau) et des modes différentiels (oscillations des cordes du doublet en opposition de phase). Le chevalet se comporte globalement comme un support résistant, mais relié à deux cordes faiblement désaccordées, il a un comportement élastique vis à vis de la corde accordée plus haut, et un comportement inerte vis à vis de l'autre corde, tendant ainsi à égaliser les fréquences de vibration des deux cordes.

\subsection{Vibrations par sympathie.}

Le phénomène de vibrations sympathiques est relativement simple. Lorsqu'une corde vibre, elle excite le chevalet à la fréquence $\mathrm{F}$ de son fondamental, mais aussi aux fréquences $2 . \mathrm{F}, 3 . \mathrm{F}, \ldots$, de ses harmoniques. Le chevalet fera donc entrer en résonance les cordes dont le fondamental est 2.F, 3.F, etc. De même le fondamental d'une corde aigüe peut exciter un partiel d'une corde grave.

\subsection{Conclusion.}

Cette étude nous a permis d'orienter nos recherches pour la modélisation de structures couplantes : nous savons désormais que les phénomènes essentiels à obtenir sont : la rémanence, les vibrations sympathiques, la disparition des battements. Pour cela nous disposons d'un indice primordial : le chevalet est un support résistant. 


\section{MODELISATION PHYSIQUE D'UNE STRUCTURE DE COUPLAGE.}

Les différentes étapes de modélisation ont été les suivantes :

- Vérification de la théorie des supports sur une seule corde.

- Mise au point d' un modèle de chevalet supportant deux cordes. Critère essentiel : la rémanence.

- Généralisation du modèle pour lui adapter $\mathrm{N}$ cordes. Critère principal : vibrations sympathiques.

4.1. Essais de supports.

Les premiers essais que nous avons effectués sur CORDIS-ANIMA étaient destinés à vérifier si la théorie des supports était valable dans le contexte de modélisation physique. Nous avons par exemple testé le système représenté figure 3 .

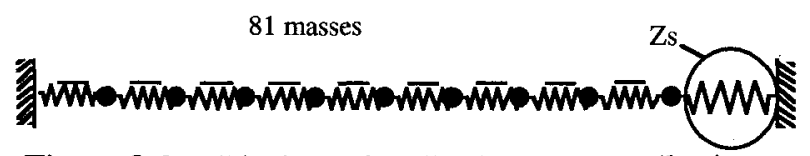

Figure 3. Modèle de corde reliée à un support élastique.

La fréquence de la corde diminue d'autant plus que la raideur d'attache est faible, conformément à la théorie. Des expériences similaires ont été concluantes avec des modèles de supports résistants et inertes. Nous pouvons donc nous baser sur le fait qu'un chevalet sera modélisable à partir de viscosités CORDIS essentiellement, puisque c'est un support résistant. Il faut cependant trouver le moyen de le relier à deux cordes.

\subsection{Modèle $\mathrm{N}^{\circ} 1$ de support pour doublet de cordes.}

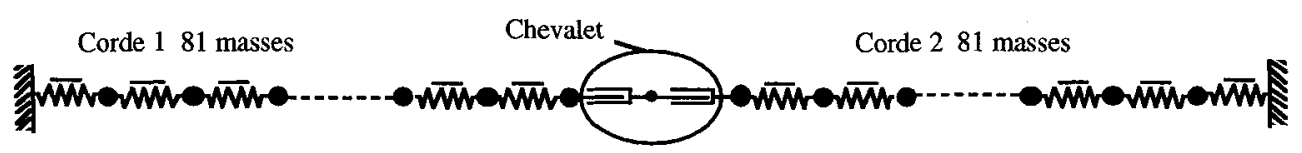

Figure 4.Le modèle de chevalet $\mathrm{N}^{\circ} 1$

Ce premier modèle de chevalet a été créé sur les bases suivantes : le chevalet est représenté par une viscosité, puisque c'est un support résistant. Il est relié à la table d'harmonie - représentée par une masse - et aux cordes. Comme il est impossible d'attacher 2 cordes et une masse à une viscosité (il n'y a que deux extrémités), il est nécessaire d'avoir deux viscosités. Ce modèle permet d'obtenir une forme de rémanence, mais on constate que, contrairement au cas réel, la rémanence vient du fait que les deux cordes entrent en phase. En effet, lorsque les masses situées de part et d'autre des viscosités sont en phase et ont la même amplitude, les viscosités ne sont plus sollicitées, le mouvement est donc moins amorti. Pour avoir rémanence, il faut que la masse de la table d'harmonie soit du même ordre que celles qui composent les cordes, contrairement au cas réel. En outre, il s'avère que ce modèle ne permet pas d'obtenir de vibrations sympathiques, car la masse de la table d'harmonie est trop faible. Ce modèle n'est donc pas réaliste.

\subsection{Modèle $\mathrm{N}^{\circ} 2$.}

En examinant le fonctionnement d'un piano réel, on constate que l'amortissement vient en fait de la table d'harmonie. Cette table est reliée au corps du piano, que l'on peut considérer comme fixe.

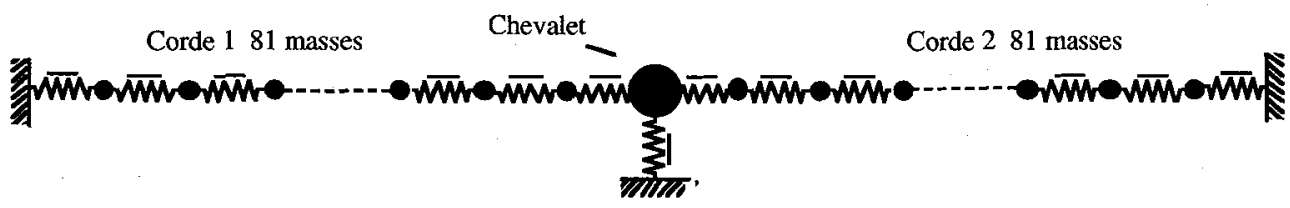

Figure 5. Modèle de chevalet $\mathrm{N}^{\circ} 2$

La masse centrale modélise le chevalet. La valeur de cette masse doit être environ mille fois la valeur des masses de corde. Avec ce modèle, la rémanence est obtenue de la même manière que dans le cas réel : il faut et il suffit d'introduire une légère différence d'amplitude à l'excitation (entre 5 et $10 \%$ ). (figure 6). On constate que les deux cordes se mettent à vibrer en opposition de phase, d'où un amortissement beaucoup plus faible que lors du son immédiat. Ce modèle reproduisant le comportement du chevalet réel pour deux cordes, nous avons décidé de le conserver pour vérifier s'il pouvait reproduire le phénomène de vibrations sympathiques. 


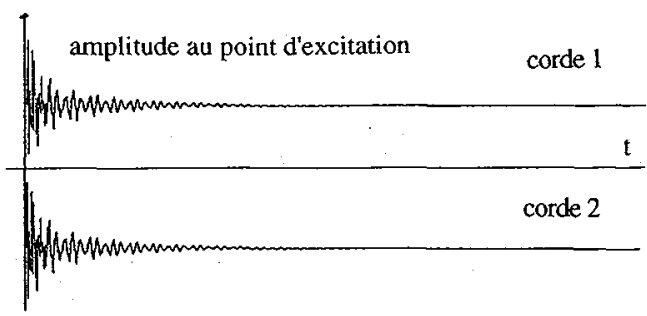

(a) excitation identique des deux cordes.

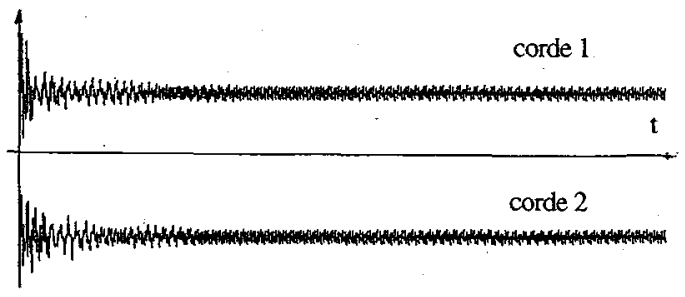

(b) écart d'amplitude d'excitation de $10 \%$.

Figure 6. Mise en évidence de la rémanence avec le modèle N².

\subsection{Généralisation du support à $N$ cordes.}

Avant d'élaborer de "gros" modèles, nécessitant des temps de calcul important, nous avons commencé par relier deux doublets de cordes au modèle $\mathrm{N}^{\circ} 2$. Les expériences visaient à vérifier les vibrations par sympathie des deux doublets. Le principe est le suivant : on accorde les deux doublets de manière harmonique, on excite l'un des deux et on observe la réponse de l'autre. Ainsi, trois types d'expériences sont possibles :

a) Les deux doublets ont le même fondamenal.

b) Le doublet percuté à un fondamental à la fréquence $\mathrm{F}$, l'autre à la fréquence $\mathrm{N}^{*} \mathrm{~F}$. Dans ce cas, le Nième harmonique du doublet percuté excite le fondamental du doublet libre.

c) Le doublet percuté à un fondamental à la fréquence $N^{*} \mathrm{~F}$, l'autre à la fréquence $\mathrm{F}$. Dans ce cas, le fondamental du doublet percuté excite le Nième harmonique du doublet libre.

Des documents vidéo ont été réalisés, visualisant les déformées des modèles, et mettant en évidence les vibrations sympathiques de deux doublets de cordes. Il était alors possible de construire des modèles plus important. Deux modèles de "piano" ont été construits. L'un où les doublets sont accordés de manière harmonique, l'autre sur la gamme tempérée. Les cordes comprennent 21 masses chacune. Pour un modèle à 40 doublets, cela représente 1680 masses en interaction, soit environ 3 heures de calcul, sur station Silicon Graphics.

Pour ces modèles, de nouveau problèmes voient le jour : où placer les points d'écoute ? Réaliser un modèle de plaque pour la table d'harmonie ? Un modèle de barre pour le chevalet? Alors intervient le problème du temps de calcul. Toujours est-il que les films video réalisés, ainsi que les sons obtenus avec les modèles complexes mettent particulièrement bien en évidence les vibrations sympathiques.

\section{PERSPECTIVES.}

Si le modèle réalisé se comporte mécaniquenent comme l'ensemble chevalet-table d'harmonie d'un piano, il reste encore de nombreux problèmes à régler si l'on veut améliorer la qualité des sons produits. Tout d'abord, les cordes CORDIS sont des modèles de cordes infiniment minces, ce qui n'est pas le cas dans la réalité. Ensuite, le modèle de chevalet reste pour l'instant très simpliste. Il faudrait étudier un modèle de plaque pour la table d'harmonie, et un modèle de barre de flexion pour le chevalet. Enfin vient le problème de la production du signal sonore par la structure vibrante ? La solution pour l'instant adoptée (point d'écoute unique et localisé) est par trop simplificatrice : si le point est mal choisi, on peut éliminer de nombreux harmoniques. Il faut bien être conscient, qu'à aucun moment on ne fait intervenir l'onde acoustique émise par l'objet vibrant. Ceci constitue le point de départ d'une nouvelle direction d'études : la modélisation de la spatialisation dans le formalisme CORDIS-ANIMA.

\section{Reférences :}

[1] Cadoz C., Luciani A. et Florens J.L., "CORDIS-ANIMA : A modeling and Simulation System for Sound and Image Synthesis - The General Formalism", Computer Music J., vol 17:1, spring 1993, S.T. Pope Ed., MIT Press, pp. 19-29.

[2] Cadoz C., Luciani A. et Florens J.L., "CORDIS-ANIMA : système de modélisation et simulation d'objets physiques".Colloque International sur les Modèles Physiques, Grenoble France septembre 1990. Ed. Maison des Sciences de l'Homme, Paris, à paraître.

[3] Weinreich G., "Coupled piano strings", J.Acoust.Soc. Am., 62 N6 (1977), 1474-1484.

[4] Bibliothèque Pour la Science, "Sons et Musique", Ed. Pour la Science S.A.R.L., Belin, Paris.

[5] Boutillon X., "Aperçu général sur les modèles physiques de piano". Colloque International sur les Modèles Physiques, Grenoble France septembre 1990. Ed. Maison des Sciences de l'Homme, Paris, à paraître.

[6] Boutillon X., "Le Piano: Modélisation physique et développements technologiques", Premier Congrès Français d'Acoustique Vol. II, P. Filippi, M. Zakharia Ed. Lyon France 1990 (Les Editions de Physique, les Ülis, 1990) pp. 811-819. 